\title{
Parameters Estimation of Three Mixed Exponential Distributions
}

\author{
G.Q. Zhang \\ Department of Mathematics and Information Science \\ North China University of Water Resources and Electric Power \\ Zhengzhou, China
}

\begin{abstract}
Mixed exponential distributions play an important role in life time data analysis, but if we use traditional statistical methods to estimate the parameters in the model, it will be very difficult, however we apply the generalized expectation maximization (GEM) algorithm, namely expectation conditional maximization (ECM) algorithm, to estimate the parameters of the model, it will greatly simplify the complexity of the calculation. In this paper, we study the parameter estimation problem in complete data situation, and give Monte Carlo (MC) simulation, which results show that the algorithm based on ECM to estimate the parameters of the mixed exponential distribution is very effective.
\end{abstract}

Keywords-mixed exponential distribution; ECM algorithm; MC simulation

\section{INTRODUCTION}

In engineering, medicine, biology and etc, life time data analysis has became a problem which statisticians and actual workers are very concerned about. There are very good statistical methods about life data analysis for a single population, but in the practical life there often are more populations. Therefore, the study of the distribution of the mixed parameter estimation will become very important. Zhu Liping, Lu Yiqiang and Mao Shisong [1,3] give estimation of parameters of the single parameter mixed exponential distribution with EM algorithm. Demester, Laird and Rubin [2, $4,5]$ put forward the EM algorithm, which greatly simplifies the calculation of maximum likelihood estimation, is recently developed quickly and is widely used, and give out the generalized EM algorithm (namely GEM) when there is no explicit format in maximizing. For multidimensional parameters, Meng and Rubin [1,6] give a special kind of GEM algorithm, which is called ECM algorithm, this algorithm retains the simplicity and stability of EM algorithm and greatly simplify the calculation in maximizing in EM algorithm. This paper gives estimation of parameter of mixed exponential distribution with the ECM algorithm in the complete data, and provides the MC simulation. Just considering the three mixed exponential distributions in the following.

Set the subpopulation $X_{k} \sim E\left(\lambda_{k}\right)(k=1,2,3)$, which probability density function is

$$
f_{k}\left(x \mid \lambda_{k}\right)=\lambda_{k} e^{-\lambda_{k} x} \quad x>0 \quad(k=1,2,3) \text { and the }
$$

population $X$ satisfies:

$$
P\left\{X=X_{1}\right\}=p, P\left\{X=X_{2}\right\}=q, P\left\{X=X_{3}\right\}=
$$

$1-p-q$, then the population $X$ submits to the three mixed exponential distributions, which probability density function is

$$
\begin{aligned}
& f(x \mid \alpha)=p \lambda_{1} e^{-\lambda_{1} x}+q \lambda_{2} e^{-\lambda_{2} x}+(1-p-q) \lambda_{3} e^{-\lambda_{3} x} \\
& \text { where } \quad \alpha=\left(p, q, \lambda_{1}, \lambda_{2}, \lambda_{3}\right) \quad, \quad 0<p, q<1, \\
& \lambda_{1}, \lambda_{2}, \lambda_{3}>0 .
\end{aligned}
$$

\section{Estimation of parameter for complete data}

Let $X_{1}, X_{2}, \cdots, X_{n}$ are the samples for the mixed exponential distributions, and $x_{1}, x_{2}, \cdots, x_{n}$ are the observed values for the samples. Let

$$
\begin{gathered}
f_{1 i}=f_{1 i}\left(x_{i} \mid \alpha\right)=\lambda_{1} e^{-\lambda_{1} x_{i}}, f_{2 i}=f_{2 i}\left(x_{i} \mid \alpha\right)=\lambda_{2} e^{-\lambda_{2} x_{i}} \\
f_{3 i}=f_{3 i}\left(x_{i} \mid \alpha\right)=\lambda_{3} e^{-\lambda_{3} x_{i}} \\
f_{i}=f_{i}\left(x_{i} \mid \alpha\right)=p f_{1 i}+q f_{2 i}+(1-p-q) f_{3 i}
\end{gathered}
$$

For random variable $Y$, which satisfies

$$
\begin{array}{cl}
P(Y=1)=p & P(Y=2)=q \\
P(Y=3)=1-p-q \text {, and } Y=k & (k=1,2,3) \text { means that }
\end{array}
$$
random variable $X_{i}$ comes from the subpopulation, which probability density function is $f_{k i}=f_{k i}\left(x_{i} \mid \alpha\right)=\lambda_{k} e^{-\lambda_{k} x_{i}}$. Then $X_{i}$ submits to the three mixed exponential distributions $X$.

So the joint distribution between $X_{i}$ and $Y$ is [7, 8]

$$
f\left(x_{i}, y \mid \alpha\right)= \begin{cases}p f_{1 i} & (y=1) \\ q f_{2 i} & (y=2) \\ (1-p-q) f_{3 i} & (y=3)\end{cases}
$$

and with the given $x_{i}$, the situation distribution for $Y$ is [7, 


$$
\begin{aligned}
& P\left(Y=1 \mid x_{i}, \alpha\right)=\frac{p f_{1 i}}{f_{i}}, \quad P\left(Y=2 \mid x_{i}, \alpha\right)=\frac{q f_{2 i}}{f_{i}}, \\
& P\left(Y=3 \mid x_{i}, \alpha\right)=\frac{(1-p-q) f_{3 i}}{f_{i}}
\end{aligned}
$$

Given the initial value $\alpha_{0}=\left(p_{0}, q_{0}, \lambda_{01}, \lambda_{02}, \lambda_{03}\right)$, the ECM algorithm is in the following.

(1) Expectation for $m=1,2, \cdots$

$$
\begin{aligned}
& Q\left(\alpha \mid \alpha_{m-1}\right)=\sum_{i=1}^{n} E_{Y}\left\{\ln f\left(x_{i}, y \mid \alpha, \alpha_{m-1}\right)\right\} \\
= & \sum_{i=1}^{n}\left\{\frac{p_{m-1} f_{(m-1) 1 i}}{f_{(m-1) i}} \ln \left(p f_{1 i}\right)+\frac{q_{m-1} f_{(m-1) 2 i}}{f_{(m-1) i}} \ln \left(q f_{2 i}\right)\right. \\
+ & \left.\left.\frac{\left(1-p_{m-1}-q_{m-1}\right) f_{(m-1) 3 i}}{f_{(m-1) i}} \ln \left[(1-p-q) f_{3 i}\right)\right]\right\}
\end{aligned}
$$$$
\left.=\sum_{i=1}^{n}\left\{c_{(m-1) 1 i} \ln \left(p f_{1 i}\right)+c_{(m-1) 2 i} \ln \left(q f_{2 i}\right)+c_{(m-1) 3 i} \ln \left[(1-p-q) f_{3 i}\right)\right]\right\}
$$$$
=\sum_{i=1}^{n}\left\{c_{(m-1) 1 i}\left[\ln p+\ln \lambda_{1}-\lambda_{1} x_{i}\right]+c_{(m-1) 2 i}\left[\ln q+\ln \lambda_{2}-\lambda_{2} x_{i}\right.\right.
$$$$
\left.+c_{(m-1) 3 i}\left[\ln (1-p-q)+\ln \lambda_{3}-\lambda_{3} x_{i}\right]\right\}
$$

Where

$$
\begin{aligned}
& \alpha_{m-1}=\left(p_{m-1}, q_{m-1}, \lambda_{(m-1) 1}, \lambda_{(m-1) 2}, \lambda_{(m-1) 3}\right), \\
& f_{(m-1) i}=f_{i}\left(x_{i} \mid \alpha_{m-1}\right), \quad f_{(m-1) 1 i}=f_{1 i}\left(x_{i} \mid \alpha_{m-1}\right) \\
& f_{(m-1) 2 i}=f_{2 i}\left(x_{i} \mid \alpha_{m-1}\right), \quad f_{(m-1) 3 i}=f_{3 i}\left(x_{i} \mid \alpha_{m-1}\right), \\
& c_{(m-1) 1 i}=\frac{p_{m-1} f_{(m-1) 1 i}}{f_{(m-1) i}}, \quad c_{(m-1) 2 i}=\frac{q_{m-1} f_{(m-1) 2 i}}{f_{(m-1) i}}, \\
& c_{(m-1) 3 i}=\frac{\left(1-p_{m-1}-q_{m-1}\right) f_{(m-1) 3 i}}{f_{(m-1) i}} . \\
& \text { (2) Maximation for } \alpha_{m}
\end{aligned}
$$$$
Q\left(\alpha_{m} \mid \alpha_{m-1}\right)=\max _{\alpha} Q\left(\alpha \mid \alpha_{m-1}\right) .
$$

(3) Let $\alpha_{m}$ the new initial value, repeat the above (1) and (2), until $\left\|\alpha_{m}-\alpha_{m-1}\right\|<\sigma$, where $\sigma$ is the given threshold value in advance, then stop iteration.

Because $Q\left(\alpha \mid \alpha_{m-1}\right)$ is a transcendental equation about $\alpha=\left(p, q, \lambda_{1}, \lambda_{2}, \lambda_{3}\right)$, it is difficult to directly solve the equation

$$
\frac{\partial Q\left(\alpha \mid \alpha_{m-1}\right)}{\partial \alpha}=0
$$

to estimate the parameter $\alpha$, and sometime it is not real. However it is easy to use the ECM algorithm and Newton iterative method.

Set $\alpha=\left(\alpha_{1}, \alpha_{2}, \cdots, \alpha_{k}\right)$, the above step (2) is decomposed by the following conditional maximation for $k$ times. Let $\alpha_{m-1}=\left(\alpha_{(m-1) 1}, \alpha_{(m-1) 2}, \cdots, \alpha_{(m-1) k}\right)$. In the $m$ step iteration, let $\alpha_{2}=\alpha_{(m-1) 2}, \cdots$, $\cdots, \alpha_{k}=\alpha_{(m-1) k}$ and solve

$$
Q\left(\alpha_{m 1} \mid \alpha_{m-1}\right)=\max _{\alpha_{1}} Q\left(\alpha \mid \alpha_{m-1}\right)
$$

then let $\alpha_{1}=\alpha_{(m-1) 1}, \alpha_{3}=\alpha_{(m-1) 3}, \cdots, \alpha_{k}=\alpha_{(m-1) k}$ and solve

$$
Q\left(\alpha_{m 2} \mid \alpha_{m-1}\right)=\max _{\alpha_{2}} Q\left(\alpha \mid \alpha_{m-1}\right)
$$

Repeat $k$ times, then complete iteration and ]$_{\text {get } \alpha_{m}}=\left(\alpha_{m 1}, \alpha_{m 2}, \cdots, \alpha_{m k}\right)$.

In the following, it gives the estimation of parameter $\alpha=\left(p, q, \lambda_{1}, \lambda_{2}, \lambda_{3}\right)$ for $k=5$.

(1) Estimating $p$ and $q$

$$
\begin{aligned}
& \frac{\partial Q\left(\alpha \mid \alpha_{m-1}\right)}{\partial p}=\sum_{i=1}^{n}\left\{c_{(m-1) 1 i} \frac{1}{p}+c_{(m-1) 3 i} \frac{-1}{1-p-q}\right\} \\
& =\sum_{i=1}^{n}\left\{\frac{(1-p-q) c_{(m-1) 1 i}-p c_{(m-1) 3 i}}{p q(1-p-q)}\right\}
\end{aligned}
$$

and

$$
\begin{aligned}
& \frac{\partial Q\left(\alpha \mid \alpha_{m-1}\right)}{\partial q}=\sum_{i=1}^{n}\left\{c_{(m-1) 2 i} \frac{1}{q}+c_{(m-1) 3 i} \frac{-1}{1-p-q}\right\} \\
& =\sum_{i=1}^{n}\left\{\frac{(1-p-q) c_{(m-1) 2 i}-q c_{(m-1) 3 i}}{q(1-p-q)}\right\}
\end{aligned}
$$

Let $\frac{\partial Q\left(\alpha \mid \alpha_{m-1}\right)}{\partial p}=0$ and $\frac{\partial Q\left(\alpha \mid \alpha_{m-1}\right)}{\partial q}=0$, then the solutions are

$$
p_{m}=\frac{\sum_{i=1}^{n} c_{(m-1) 1 i}}{n} \text { and } q_{m}=\frac{\sum_{i=1}^{n} c_{(m-1) 1 i}}{n} .
$$


(2) Estimating $\lambda_{1}, \lambda_{2}, \lambda_{3}$

$$
\frac{\partial Q\left(\alpha \mid \alpha_{m-1}\right)}{\partial \lambda_{k}}=\sum_{i=1}^{n}\left\{c_{(m-1) 1 i}\left(\frac{1}{\lambda_{k}}-x_{i}\right)\right\} \quad(k=1,2,3)
$$

Let $\frac{\partial Q\left(\alpha \mid \alpha_{m-1}\right)}{\partial \lambda_{k}}=0$, then the solutions are

$$
\lambda_{m 1}=\frac{\sum_{i=1}^{n} c_{(m-1) 1 i}}{\sum_{i=1}^{n} c_{(m-1) 1 i} x_{i}}
$$

$$
\lambda_{m 2}=\frac{\sum_{i=1}^{n} c_{(m-1) 2 i}}{\sum_{i=1}^{n} c_{(m-1) 2 i} x_{i}}, \lambda_{m 3}=\frac{\sum_{i=1}^{n} c_{(m-1) 3 i}}{\sum_{i=1}^{n} c_{(m-1) 3 i} x_{i}} .
$$

III. MC Simulations and Analysis

Let the real value $p=0.3, q=0.4, \lambda_{1}=1$, $\lambda_{2}=0.6, \lambda_{3}=0.2 \quad, \quad$ the initial value $p_{0}=0.2$, , $q_{0}=0.3, \lambda_{01}=0.8, \lambda_{02}=0.3, \lambda_{03}=0.1$ and the sample size, then give MC simulation tests for 1000 times

TABLE I. Estimations of Parameters for MEAN and MSE.

\begin{tabular}{|l|l|l|l|l|l|}
\hline & & $n=40$ & $n=60$ & $n=80$ & $n=100$ \\
\hline \multirow{3}{*}{$p$} & MEAN & $2.85 \mathrm{E}-01$ & $3.04 \mathrm{E}-01$ & $2.95 \mathrm{E}-01$ & $2.98 \mathrm{E}-01$ \\
\cline { 2 - 6 } & MSE & $1.42 \mathrm{E}-02$ & $1.39 \mathrm{E}-02$ & $1.30 \mathrm{E}-02$ & $1.12 \mathrm{E}-02$ \\
\hline \multirow{3}{*}{$q$} & MEAN & $3.89 \mathrm{E}-01$ & $3.95 \mathrm{E}-01$ & $4.08 \mathrm{E}-01$ & $3.99 \mathrm{E}-01$ \\
\cline { 2 - 6 } & MSE & $1.48 \mathrm{E}-02$ & $1.30 \mathrm{E}-02$ & $1.35 \mathrm{E}-02$ & $1.18 \mathrm{E}-02$ \\
\hline \multirow{2}{*}{$\lambda_{1}$} & MEAN & $9.85 \mathrm{E}-01$ & $9.90 \mathrm{E}-01$ & $9.95 \mathrm{E}-01$ & $9.98 \mathrm{E}-01$ \\
\cline { 2 - 6 } & MSE & $1.86 \mathrm{E} 00$ & $1.71 \mathrm{E} 00$ & $1.60 \mathrm{E} 00$ & $1.58 \mathrm{E} 00$ \\
\hline$\lambda_{2}$ & MEAN & $5.85 \mathrm{E}-01$ & $5.91 \mathrm{E}-01$ & $6.05 \mathrm{E}-01$ & $6.01 \mathrm{E}-01$ \\
\cline { 2 - 6 } & MSE & $9.96 \mathrm{E}-01$ & $8.85 \mathrm{E}-01$ & $7.79 \mathrm{E}-01$ & $6.68 \mathrm{E}-01$ \\
\hline \multirow{2}{*}{$\lambda_{3}$} & MEAN & $1.86 \mathrm{E}-01$ & $2.03 \mathrm{E}-01$ & $1.96 \mathrm{E}-01$ & $2.01 \mathrm{E}-01$ \\
\cline { 2 - 6 } & MSE & $5.56 \mathrm{E}-01$ & $5.51 \mathrm{E}-01$ & $4.45 \mathrm{E}-01$ & $4.42 \mathrm{E}-01$ \\
\hline
\end{tabular}

NOTES: MEAN FOR MEAN AND MSE FOR MEAN SQUARE ERROR OF THE SAMPLES.

Table 1 indicates that it is good to use the ECM algorithm to estimate the parameters for the three mixed exponential distribution. The estimation of the parameters is all very close to the real values of the parameter $\alpha$. At the same time, the MSE values of the samples are more and smaller along with the increase of the sample size $n$. So the method can be regarded as a kind of very effective statistical calculation method.

\section{REFERENCES}

[1] Zhu Liping, Lu Yiqiang and Mao Shisong, Estimation of Parameters of Mixed Exponential Distribution, Chinese Journal of Applied Probability and Statistics, Vol. 22(2), 2006, pp:137-150.

[2] Demester A, Laird N M and Rubin D B, Maximum likelihood from incomplete data via EM algorithm, Journal of Royal Statistical Society, Series B, Vol. 39(1), 1977, pp:1-38.

[3] Mao Shisong,Wang Jinglong and Pu Xiaolong, Higher mathematical statistics, Beijing: Higher education press, 1998.

[4] Aitkin M and Wilson G T, Mixture models, outliers and the EM algorithm, Technometrics, Vol. 22, 1980, pp: 325-331.

[5] Dickinson J P, On the resolution of a mixture of observations from two gamma distributions by the method of maximum likelihood, Metrika, Vol. 21, 1974, pp: 133-141.

[6] Falls L W, Estimation of parameters in compound Weibull distributions, Techno-metrics, Vol.12, 1970, pp: 339-407.

[7] Folks E B, Some method for studying the mixture of two normal distributions, Journal of American Statistics Association, Vol.74, 1979, pp:561-575.
[8] Jiang Hui and Tang Yincai, Estimation of Parameters of Mixed Weibull Distributions, Chinese Journal of Systems Science and Mathematical Sciences, Vol.30(1), 2010, pp:79-88. 